\title{
Xenografts of Pediatric Solid Tumors: Predictive Intermediate Models?*
}

\author{
P.J. Houghton and J.A. Houghton
}

\section{A. Introduction}

Developing new therapy for treatment of solid tumors of childhood presents certain problems that are not encountered frequently with adult malignancies. In most instances, new agents are discovered through a process of serendipity, and certainly, design based upon metabolic characteristics of pediatric tumors has not been a major focus. Compounds showing potential against predominantly murine tumors and eliciting acceptable toxicity in preclinical toxicologic evaluations progress to traditional phase-I and phase-II clinical evaluation in adults. In the United States it is rare that a new anticancer drug will be tested in children simultaneously, or before adult phase-I trials have been completed. At this point in development, the new agent may fail to stimulate sufficient enthusiasm to further its evaluation in childhood malignancies and the compound may be discarded. However, there is no reason to assume that a failure to show activity against certain human tumors, predominantly of epithelial origin, will translate into a similar finding with childhood tumors of different origin. In fact, less than one in three new drugs evaluated in adults have received adequate testing

Laboratories for Developmental Therapeutics, Dept. Biochemical and Clinical Pharmacology St. Jude Children's Research Hospital, Memphis, TN 38101, USA

* This work was supported in part by PHS awards CA 38933, CA 23099, CA 32613, CH423 from the American Cancer Society and by the American Lebanese Syrian Associated Charities against childhood malignancies. In part, this is a consequence of there being relatively few patients, precluding large-scale evaluation in randomized trials.

A second problem is that phase-II evaluation is conducted in children who have been exposed to multiple chemotherapeutic agents and have clearly resistant tumors. In addition, these patients can usually tolerate only reduced dose levels of a drug. Thus, a failure to demonstrate activity in these patients does not necessarily mean that the drug may not be active against the same tumor at diagnosis. However, evaluating new agents in previously untreated patients is in most cases not possible for childhood malignancies, because for many tumor types there is available, effective, and sometimes curative therapy. Thus, we find ourselves in a dilemma: clearly, we require new effective agents, but traditional means for identifying these are inadequate due to the reasons already given.

With this perspective we have approached the problem in the following manner. Our laboratories have been involved in developing preclinical models with the specific aim of ultimately developing histiotype-specific therapy. That is, instead of studying "generic cancer," we believe that new agents can be identified based upon the metabolic characteristics of particular histiotypes, or subtypes. The first step in testing such a hypothesis has been to develop specific models and to determine the relevance of these with respect to biologic, metabolic, and chemosensitivity characteristics. The approach we have taken is to establish human tumors in immune-deprived 
mice. The models that will be dealt with here are rhabdomyosarcomas (RMS) of children and adult colon adenocarcinomas. These two types were chosen because they show quite different chemosensitivities and hence are of value for determining the relevance of this approach. In addition, we have established xenografts of rhabdomyosarcomas at relapse. Clearly, for the model to be of use these tumors should be significantly less sensitive than their counterparts heterotransplanted at diagnosis.

Results obtained with these models indicate that when used intelligently, human tumor xenografts may have a significant role in identifying agents that should receive priority for evaluation in childhood malignancies, and such data may be used further to justify evaluation of an agent in previously untreated children (with poor prognosis), even when it fails to demonstrate significant activity in classical phase-II evaluation against relapse tumors.

\section{B. Material and Methods}

\section{Immune-deprived Mice}

Four-week-old female CBA/CaJ mice were immune-deprived by thymectomy, followed at 3 weeks by total body irradiation (TBI; $925 \mathrm{cGy},{ }^{137} \mathrm{Cs}$ source). Mice received either cytosine arabinoside $(200 \mathrm{mg} / \mathrm{kg}) 48 \mathrm{~h}$ prior to TBI, or $3 \times 10^{6}$ nucleated marrow cells within $6 \mathrm{~h}$ of TBI $[1,2]$. Mice were housed under conventional conditions in a humidity- and temperature-controlled environment, as described previously $[1,2]$.

\section{Tumor Lines and Characteristics}

Three models were used. Human colon adenocarcinomas were established from untreated primary lesions as described previously $[2,3]$. These tumors retain morphologic, histologic, and karyotypic characteristics when grown in mice. For the first model of childhood RMS, lines were established from untreated tumor material, either primary or metastatic lesions. Characteristics of these tumors, histology, and karyotype have been presented in detail $[1,4]$. In addition, five lines of RMS were established from patients with clearly progressive disease [5].

\section{Experimental Design}

Tumor material was transplanted into mice 2 weeks after TBI. Mice received a single administration of agent when the mean tumor diameter was $>1 \mathrm{~cm}$. Diameters were measured at 7-day intervals, and volumes were calculated as described previously [4]. Response criteria are presented in Table 1.

\section{Results}

The initial question that we posed was whether xenografts accurately represented the chemosensitivity of the tumor type from which they were derived. For this experiment we constructed three models, each comprising five or six tumor lines from untreated RMS, RMS at relapse, or adult colon adenocarcinoma. Colon adenocarcinoma xenografts were chosen, as this tumor type in man is essentially refractory to all conventional chemotherapeutic agents.

The responses of colon adenocarcinoma xenografts to single maximal tolerated dose (MTD) levels of seven agents are presented in Table 1. Clearly, these tumors in mice were poorly sensitive, with only methylCCNU, 5-fluorouracil, and cyclophosphamide showing marginal activity. Thus, the overall responsiveness of these tumors parallels the lack of response observed clinically.

In contrast, RMS at diagnosis is a relatively chemosensitive tumor, and this sensitivity is paralleled when these tumors are heterografted in immune-deprived mice. Responses to the MTD for known, clinically efficacious agents are presented in Table 2. Of particular note is the marked activity of vincristine, which at this dose level caused complete regres- 


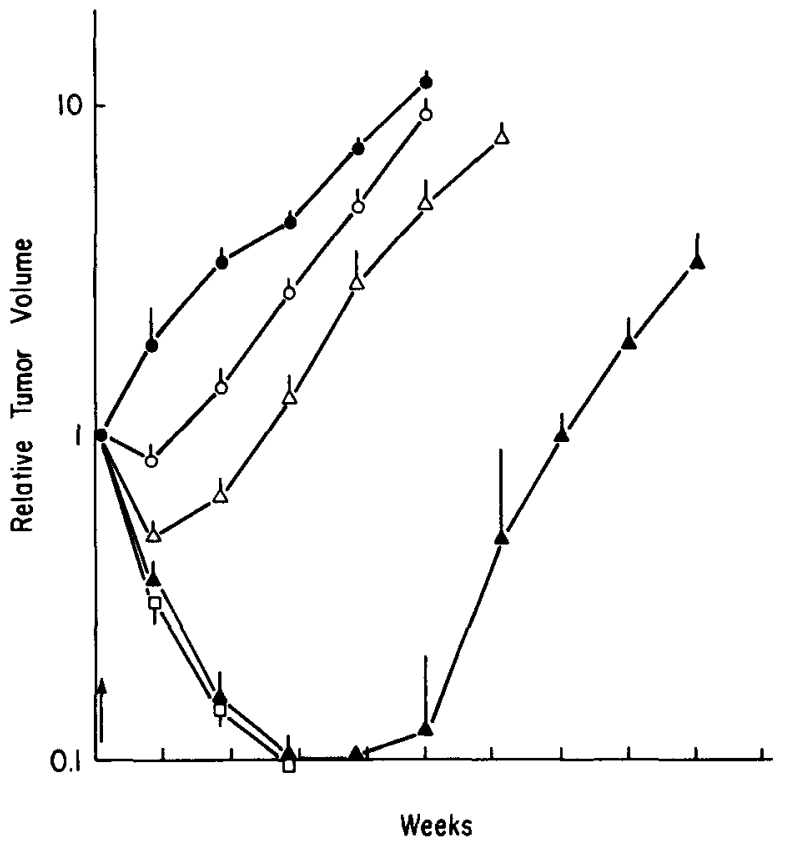

Fig. 1. Response of Rh12, childhood RMS, to increasing dose levels of vincristine (VCR). Mice received a single administration of VCR when tumors were $\geq 1 \mathrm{~cm}^{3}$. Each curve represents the mean for 14 tumors. Ordinate, tumor volume relative to that at treatment; abscissa, time after treatment $(\bullet)$ No treatment control;

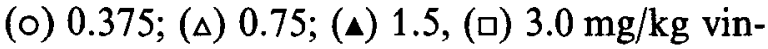
cristine

Table 4. Responses of childhood RMS: relapse

\begin{tabular}{lllll}
\hline Agent/tumor & RD & LL & CD & Rh10 \\
\hline Vincristine & ++ & + & \pm & + \\
Cyclophosphamide & + & + & + & - \\
Actinomycin D & - & - & - & - \\
Doxorubicin & - & \pm & \pm & - \\
\hline
\end{tabular}

Response criteria as for Table 1
However, it was possible that the response of RMS xenografts was a consequence of host environment. Consequently, we established RMS lines from patients at relapse [5]. The responsiveness of these tumors to the standard clinically active agents is presented in Table 4 . These tumors were significantly less sensitive to treatment than were the diagnosis specimens. These data therefore support the model as retaining chemosensitivity typical of the tumors from which it was derived.

Data derived from these models suggested that the RMS model for previously untreated tumors may be of value in identifying effective agents that had not been evaluated against this histiotype. Results with mitomycin C, cis-dichlorodiamminoplatinum, DTIC, and L-phenylalanine mustard (L-PAM) are presented in Table 5 and Fig. 2 [4]. Of these agents L-PAM showed very significant activity against five of six lines, causing complete regression of advanced tumor in four lines. Further, L-PAM had a fairly wide dose-response relationship [6].

Based on these data, a phase-I/II trial was initiated [7]. Initial studies showed that the MTD was $35 \mathrm{mg} / \mathrm{m}^{2}$ given every 21 days. Pharmacokinetic profiles for $\mathrm{L}$ PAM in children and in mice (Fig. 3) demonstrated that the area under concentration curve (AUC) and clearance were similar (Table 6). However, of 13 patients treated only one showed an objective response (Table 7). The difference between the clinical outcome and that predicted by the model (for diagnosis

Table 5. Responsiveness of xenografts of childhood rhabdomyosarcoma to DNA-reacting agents $^{\mathrm{a}}$

\begin{tabular}{lllllll}
\hline Agent/tumor & $\mathrm{H} \times \mathrm{Rh} 12$ & $\mathrm{H} \times \mathrm{Rh} 18$ & $\mathrm{H} \times \mathrm{Rh} 28$ & $\mathrm{H} \times \mathrm{Rh} 30$ & $\mathrm{H} \times \mathrm{Rh} 35$ & $\mathrm{H} \times \mathrm{Rh} 39$ \\
\hline L-PAM & +++++ & +++ & ++++++ & +++++ & ++++++ & ++++++ \\
Cyclophosphamide & ++ & +++ & ++++ & ++ & + & ++++ \\
cis-DDP & + & ++ & ++ & + & + & ++ \\
Mitomycin C & - & + & ++++ & + & ++++ & +++++ \\
DTIC & + & +++ & +++++ & ++++ & +++++ & +++ \\
\hline
\end{tabular}

a Agents administered as a single i.p. injection at equitoxic doses 


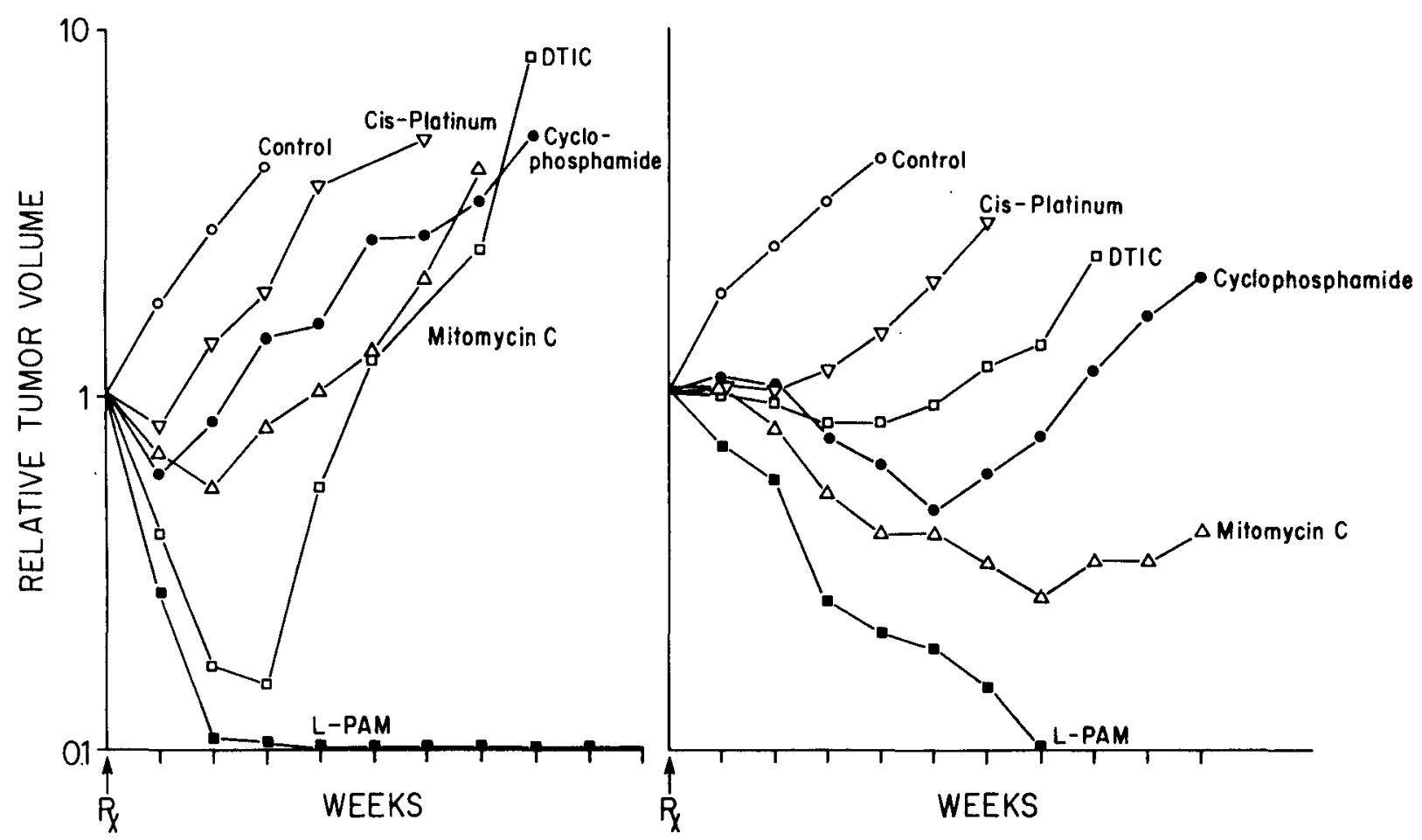

Fig. 2. Responses of Rh28 (left) and Rh39 (right) to DNA-interacting agents administered at the MTD. (From Houghton et al. [4])

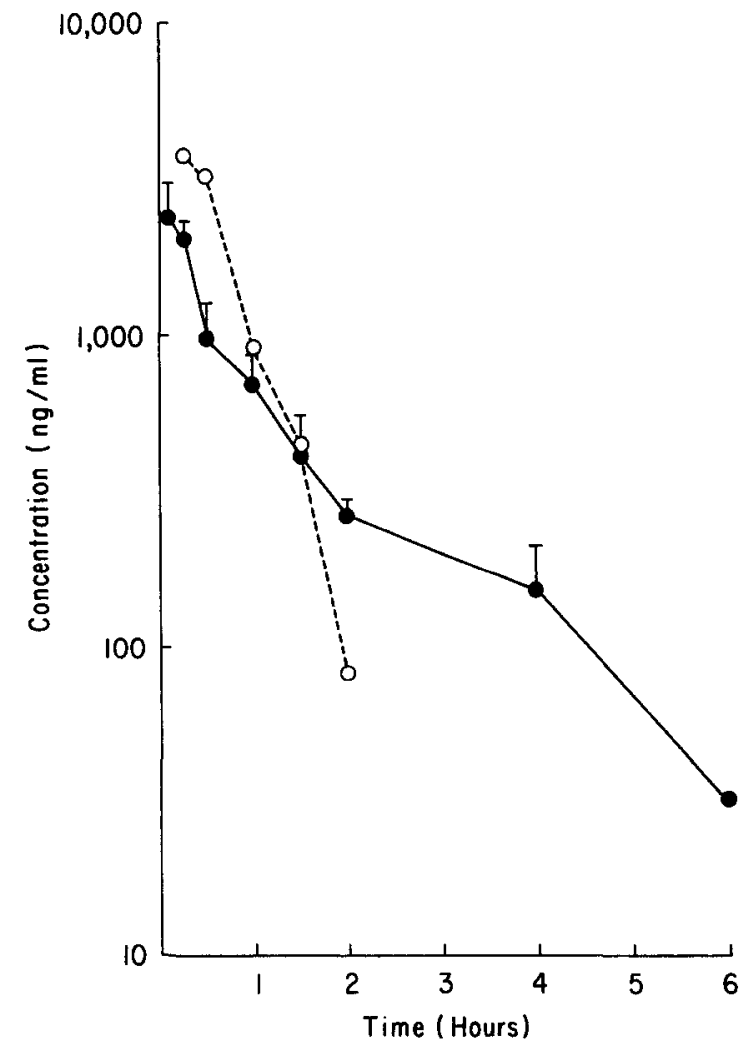

Fig. 3. Pharmacokinetic analysis of L-PAM in children and mice. Mice received $43 \mathrm{mg} / \mathrm{m}^{2}$ as a single i.p. administration (o) and children received $35 \mathrm{mg} / \mathrm{m}^{2}$ i.v. (•). Bars indicate standard deviation from the mean. (From Horowitz et al. [7])
Table 6. Pharmacokinetic parameters for $\mathrm{L}$ phenylalanine mustard

\begin{tabular}{lll}
\hline & Child & Mouse \\
\hline AUC & $175000 \mu \mathrm{g} / 1 / \mathrm{min}^{2}$ & $170000 \mu \mathrm{g} / \mathrm{l} / \mathrm{min}$ \\
Clearance & $232 \mathrm{mg} / \mathrm{min} / \mathrm{m}^{2}$ & $235 \mathrm{ml} / \mathrm{min} / \mathrm{m}^{2}$ \\
\hline
\end{tabular}

RMS) was probably not due to different pharmacokinetics, as shown in Table 6, but was probably related to evaluation of L-PAM in relapse patients with drug-resistant tumors.

To test this hypothesis, a second clinical trial was undertaken in which L-PAM $\left(45 \mathrm{mg} / \mathrm{m}^{2}\right)$ was administered to children diagnosed as having stage-4 (disseminated unresectable) RMS, for whom the prognosis was very poor. These patients have $<15 \%$ long-term survival with conventional treatment. For these patients L-PAM demonstrated very significant activity, as shown in Table 7 . 
Table 7. Clinical features of 26 children with rhabdomyosarcoma who were treated with melphalan

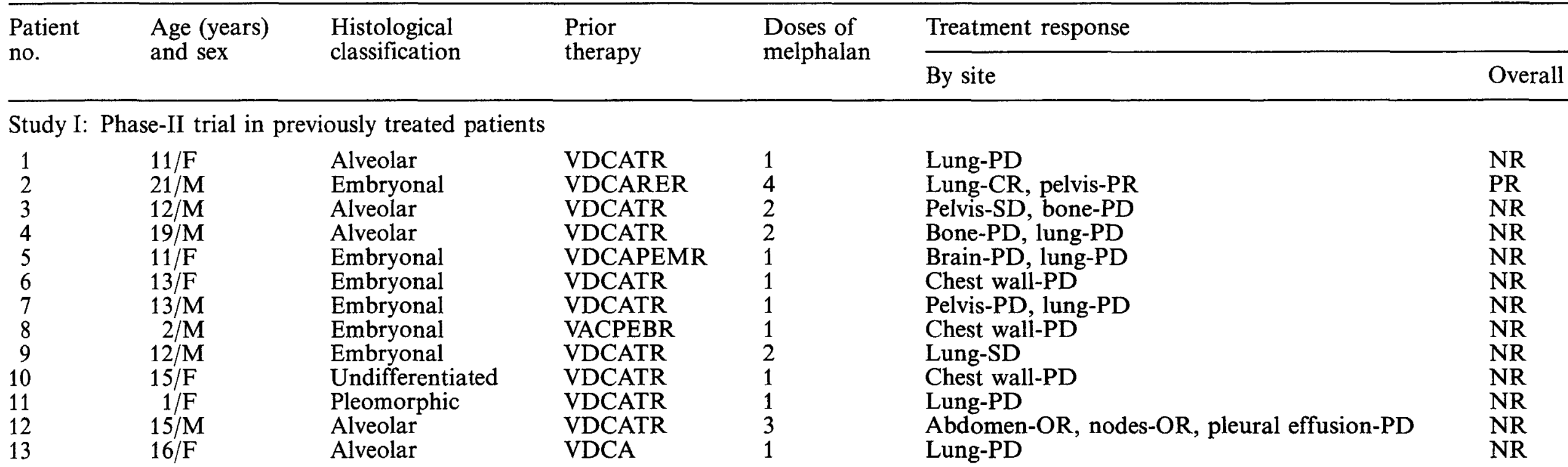

Study II: Phase-II trial in newly diagnosed patients

\begin{tabular}{lclllll}
14 & $14 / \mathrm{M}$ & Alveolar & None & 2 & \multicolumn{2}{l}{ Hand-PR, extremity-CR, abdomen-CR } \\
15 & $<1 / \mathrm{F}$ & Undifferentiated & None & 2 & Extremity-PR, nodules-CR & PR \\
16 & $7 / \mathrm{F}$ & Embryonal & None & 2 & Head-PR & PR \\
17 & $8 / \mathrm{F}$ & Embryonal & None & 2 & Head-OR, lung-PD & NR \\
18 & $4 / \mathrm{F}$ & Embryonal & None & 2 & Pelvis-PR, pleural effusion-CR & PR \\
19 & $5 / \mathrm{M}$ & Embryonal & None & 2 & Head-PR & PR \\
20 & $2 / \mathrm{M}$ & Embryonal & None & 2 & Prostata-PR & PR \\
21 & $19 / \mathrm{F}$ & Alveolar & None & 2 & Pelvis-PR, CSF-CR bone marrow-CR \\
22 & $9 / \mathrm{F}$ & Embryonal & None & 2 & Head-OR & PR \\
23 & $7 / \mathrm{F}$ & Embryonal & None & 2 & Head-PR & NR \\
24 & $3 / \mathrm{M}$ & Alveolar & None & 1 & Lung-SD & PR \\
25 & $14 / \mathrm{F}$ & Alveolar & None & 2 & Extremity-PR, bone-PR \\
26 & $3 / \mathrm{M}$ & Embryonal & None & 2 & Head-PR & PR \\
\hline
\end{tabular}

Abbreviations: A, Doxorubicin; V, vincristine; D, dactinomycin; C, cyclophosphamide; P, cisplatin; E, etoposide; T, dacarbazine; M, methotrexate; $\mathrm{B}$, dibromodulcitol; $\mathrm{R}$, radiotherapy, OR, objective response; SD, stable disease; PD, progressive disease; PR, partial response; NR, no response; $\mathrm{CR}$, complete response; CSF, cerebrospinal fluid 


\section{Discussion}

The use of human tumor xenografts is now being accepted as a means by which phase-II preclinical evaluation may be used to prioritize and direct clinical testing of new agents. This may have considerable impact on the identification of new treatments for relatively rare tumors in adults and for most solid tumors of childhood. Extensive data from a series of models suggest that the following criteria may be useful in these studies: (a) models should be comprehensive and should attempt to encompass some of the heterogeneity observed in the clinical disease. We have routinely used six independently derived lines for each model, and this appears adequate; (b) response criteria should be similar to those used clinically, i. e., $50 \%$ tumor regression would be required at the MTD for a given agent; (c) to justify further evaluation of an agent in previously untreated, poorprognosis patients, the agent should have demonstrated activity similar or superior to the most effective agent used in that disease, and in phase-I trials should have shown similar AUC and clearance to that in mice.

For most agents being evaluated in children, pharmacokinetic data from adults are often available. Thus, it is relatively easy to determine whether the mouse pharmacokinetics are similar to those that may be anticipated in children. Consequently, an agent that is tolerated in mice at dose levels that would be clearly toxic in man can be eliminated prior to trial in children. A more difficult decision is to move an "unknown" compound from the xenograft testing phase into a clinical trial. Under these conditions human tolerance has to be defined, and clearly, the mouse model may either overpredict or underpredict for efficacy in human beings. However, the criteria discussed above appear valid in this situation as well.

Acknowledgments. We thank Marc Horowitz, M.D., and Michael Christensen, Pharm. D., for fruitful collaboration.

\section{References}

1. Houghton JA, Houghton PJ, Webber BL (1982) Growth and characterization of childhood rhabdomyosarcomas as xenografts. JNCI 68:437-443

2. Houghton JA, Houghton PJ (1980) On the mechanism of cytotoxicity of fluorinated pyrimidines in four human colon adenocarcinoma xenografts maintained in immunedeprived mice. Cancer 45: 1159-1167

3. Houghton JA, Taylor DM (1978) Maintenance of biological and biochemical characteristics of human colorectal tumours during serial passage in immune-deprived mice. Br J Cancer 37:199-212

4. Houghton JA, Cook RL, Lutz PJ, Houghton PJ (1984) Childhood rhabdomyosarcoma xenografts: response to DNA-interacting agents and agents used in current clinical therapy. Eur J Cancer Clin Oncol 20:955-960

5. Houghton JA, Houghton PJ, Green AA (1982) Chemotherapy of childhood rhabdomyosarcomas growing as xenografts in immune-deprived mice. Cancer Res 42: 535-539

6. Houghton JA, Cook RL, Lutz PJ, Houghton PJ (1985) L-Phenyl-alanine mustard (NSC 8806): a potential new agent in the treatment of childhood rhabdomyosarcoma. Cancer Treat Rep 69:91-96

7. Horowitz ME, Etcubanas E, Christensen M, Houghton JA, George SL, Green AA, Houghton PJ (1988) Predictability of pediatric rhabdomyosarcoma xenografts for melphalan activity in previously untreated patients: a model for development of cancer therapy. J Clin Oncol 6:308-314 